\title{
Highlights from the issue
}

\section{Pre-hospital blood?}

Trauma transfusion protocols have changed dramatically in recent years with the early use of blood products a significant change from the bad old ATLS days of $2 \mathrm{~L}$ of crystalloid, but what of the patient in the prehospital environment? This month we publish a paper from Brisbane, Australia that examines the feasibility of prehospital blood use for ground based response services which suggests that it is feasible and practical for ground response teams.

\section{Lost in translation}

Medical publishing is dominated by the English language with the majority of journals and articles possessing an anglophile voice, but this may present problems when research findings are translated into local practice. Jobe et al in Belgium identified difficulties in the translation of English triage scales into French. This is an interesting perspective and a lesson to all who wish to translate research findings across populations with a different language base. The findings on their ELISA designed scale demonstrate an association between triage category and resource use, but does it better than translated methods? I'm not sure though the question and rationale raised by this paper is worthy of consideration.

\section{Do we really need to $\mathrm{CT}$ everything?}

The use of whole body CT (WBCT) has emerged as a key process in the management of trauma patients. In the UK it is used as a key performance indicator for trauma services and as a result more scans are being performed. The initial enthusiasm for WBCT has begun to wane in recent months as concerns about the radiation dose have been raised. Questions about the long term effects, particularly in the young, have led to calls for the use of selective areas scanning at the discretion of trauma teams, though concerns have also been raised that this will lead to missed injuries and/or deaths. Healy et al have tackled this topic in a systematic review to bring us up to date on where the evidence stands to date. The data is interesting and you really should have a look at the Forest plots to get a feeling for the strength of the evidence for WBCT. I agree with the authors, there is more to learn in this area with the question of WBCT for everyone still in some doubt.

\section{Should all trauma centres have helipads?}

A short and snappy article from a major trauma centre at Southampton in the UK demonstrates the need for a helipad to receive patients with major trauma. It's no surprise to find that more patients can access specialist trauma care if you have access to a co-located helipad, so why do many major trauma centres lack a helipad? This is further evidence for any MTCs pushing for a new helipad.

\section{Emergency medicine flow-it's a team sport}

EDs around the world are under pressure to improve flow and reduce overcrowding. Much work has focused on the work within the ED, but EDs are not islands of care, rather they work in a larger emergency care system which has significant effects on flow and resource use. Brick et al from Canada have examined the influence of speciality consultations on ED efficiency and have identified speciality consults as a process that significantly contributes to ED length of stay. Emergency medicine is a team sport and if a department is to perform then timely speciality consults are a must.

\section{Wounds, risks and time}

I was taught (and probably still teach) that old wounds should not be closed, but is this dogma or evidence based? I do like papers that challenge traditions and a paper by Quinn et al from San Francisco followed up 2663 patients with traumatic wounds looking at the risks of infection. Interestingly they did not find an association between length of time and wound infection. Perhaps we need to think again and look to other factors to assess risk with the authors identifying four other factors associated with wound infection.

\section{Does BMI predict the effectiveness of morphine in the ED?}

For those of us who work in adult and paediatric practice the difference in dosage regimes between kids (everything by weight) and adults (one size fits all) is perplexing. In paediatrics the argument is that children come in different shapes and sizes..., but any visit to an ED waiting room will demonstrate the same in adults and so it reasonable to ask whether we should adjust adult dosages against weight. Patanwala have looked at this through the effectiveness of fixed dose morphine regimes for ED patients in pain, and surprisingly to me, there is little or no difference in the effectiveness of morphine effectiveness against BMI. Good news for current pain protocols, but we cannot assume that the same is true for other drugs and with an increasingly obese population do we need more studies like this?

\section{Fractured neck of femur=perform a nerve block}

Mittal and Vermani give us a convincing argument for the use of femoral nerve blocks for our patients with fractured neck of femurs. If you are not doing it, then you should be. This paper tells you how and why. Essential reading for all EDs.

\section{Croup and climate}

A real multinational paper on the incidence of croup in Australia with authors from Sydney, Cambridge and New Brunswick looking at the climatic influence on croup attendances to the ED. In summary milder weather means fewer admissions even in the rather lovely weather in New South Wales. The authors consider such epidemiological information could be used for emergency planning? 